\title{
ON GENERATING FREE PRODUCTS OF LATTICES
}

\author{
G. GRÄTZER AND J. SICHLER ${ }^{1}$
}

ABSTRACT. For a lattice $K$ let $g(K)$ denote the cardinality of the smallest generating set of $K$. Let $L$ be the free product of the lattices $A$ and $B$. It is proved that $g(L)=g(A)+g(B)$. This is proved, in fact, for free products with respect to any given equational class of lattices. Some applications and generalizations are also given.

1. For a lattice $L$ let $g(L)$ denote the smallest cardinal $m$ such that $L$ has a generating set of cardinality $m$. In this note we compute $g(L)$ for a free product.

Theorem. Let $L$ be the free product of $A$ and $B$. Then

$$
g(L)=g(A)+g(B) .
$$

In fact, we prove this result for free $\mathbf{K}$-products, that is, for free products in an arbitrary nontrivial equational class $\mathbf{K}$.

Among the consequences of this result is that if $L$ is $n$-generated ( $n$ is a positive integer), then $L$ cannot be represented as a free product of more than $n$ lattices and, furthermore, $L$ is a free product of $n$ lattices iff $L$ is the free lattice on $n$ generators.

The theorem is proved in $\$ 2$. Some consequences of the theorem are given in $\$ 3$, while some generalizations are discussed in $\$ 4$. We refer the reader to [1] for notation and for concepts not defined in this note.

2. Let $\mathbf{K}$ be an equational class of lattices; let $\mathbf{K}$ be nontrivial, that is, let $\mathbf{K}$ contain at least one lattice with more than one element.

Let $L \in \mathbf{K}$; let $A$ and $B$ be sublattices of $L . L$ is called a free $\mathbf{K}$ product of $A$ and $B$ iff $A \cup B$ generates $L$ and for any lattice $C \in \mathbf{K}$ and lattice homomorphisms $\phi_{A}$ and $\phi_{B}$ of $A$ and $B$, respectively, into $C$, there exists a homomorphism $\phi$ of $L$ into $C$ such that $\phi$ extends both $\phi_{A}$ and $\phi_{B}$. In this section, let $L$ be a free $\mathrm{K}$-product of $A$ and $B$.

Presented to the Society, January 16, 1974; received by the editors September 10, 1973. AMS (MOS) subject classifications (1970). Primary 06A20.

Key words and phrases. Lattice, free product, generating set.

1 Research of both authors was supported by the National Research Council of Can ada. 
Let $L^{b}$ denote $L$ with a new 0 and 1 adjoined to $L$. Let $\psi$ be the common extension of the identity map on $A$ and of $B \rightarrow 0$; for $x \in L$ we denote by $x_{A}$ the image of $x$ under $\psi$ and we call $x_{A}$ the lower A-cover. Observe that $x_{A} \in A \cup\{0\} ; x \geq x_{A}$, and if $y \in A \cup\{0\}, y \leq x$, then $y \leq x_{A}$. We define similarly $x_{B}$, and dually $x^{A}(\in A \cup\{1\})$ and $x^{B}$. (See [4] or [2].)

In [3] we proved the following result.

Lemma 1. Let $X$ be a generating set of L. Then

$$
T_{A}(X)=\left\{x_{A} \mid x \in X, x_{A} \neq 0\right\}
$$

is a generating set of $A$.

By duality,

$$
T^{A}(X)=\left\{x^{A} \mid x \in X, x^{A} \neq 1\right\}
$$

is a generating set of $A$.

Recall, that if $x_{A} \in A$, then $x^{B}=1$; if not, then $x^{B} \in B$, and $x_{A} \leq x^{B}$, a contradiction since elements of $A$ and $B$ are incomparable. Similarly, if $x^{A} \in A$, then $x_{B}=0$. Finally, recall [2], [4] that if $x^{B}=1$, then $x_{A} \in A$; analogously for all the other cases. This implies that $L$ has a representation as a disjoint union

$$
L=A \cup B \cup L_{1} \cup L_{2}
$$

where

$$
\begin{aligned}
L_{1}=\left\{x \mid x \in L, x \notin A \cup B \text { and } x_{A} \in A, x_{B}=0 \text { or } x_{B} \in B, x_{A}=0,\right. & \text { or } \left.x^{A} \in A, x^{B}=1 \text { or } x^{B} \in B, x^{A}=1\right\} \\
= & \left\{x \mid x \in L, x \notin A \cup B \text { and } x_{A}, x^{A} \in A \text { or } x_{B}, x^{B} \in B\right\}, \\
L_{2} & =\left\{x \mid x \in L \text { and } x_{A} \in A, x_{B} \in B \text { or } x^{A} \in A, x^{B} \in B\right\} .
\end{aligned}
$$

The next three lemmas describe how a generating set of $L$ can be transformed into a generating set contained in $A \cup B$ with no increase of its size.

Lemma 2. Let $X$ be a generating set of $L$ and let $x \in X \cap L_{1}$. Then there is an $\bar{x} \in A \cup B$ such that $T_{1}=(X-\{x\}) \cup\{\bar{x}\}$ is a generating set of $L$, and $|X| \geq\left|T_{1}\right|$.

Proof. $x \in L_{1}$ so let, e.g., $x_{A} \in A$ and $x_{B}=0$. We set $\bar{x}=x_{A}$. To 
show that $T_{1}$ generates $L$ it is sufficient to show that $T_{1}$ generates any $a \in A$ and $b \in B$. Let $a \in A$; since $X$ generates $L, a=p\left(x, x_{1}, \cdots, x_{n}\right)$, $x_{1}, \cdots, x_{n} \in X$, for a suitable polynomial $p$. Then, since $p$ is isotone,

$$
\begin{aligned}
a=p\left(x, x_{1}, \cdots, x_{n}\right) & \geq p\left(x_{A}, x_{1}, \cdots, x_{n}\right) \\
& \geq p\left(x_{A},\left(x_{1}\right), \cdots,\left(x_{n}\right)_{A}\right)=a_{A}=a,
\end{aligned}
$$

and therefore $a=p\left(x_{A}, x_{1}, \cdots, x_{n}\right)$, as claimed.

Now let $b \in B$. Then $b=q\left(x, x_{1}, \cdots, x_{n}\right)$. Recall that, by assumption, $x_{B}=0$ and so $x \geq x_{A} \geq x_{B}$. Since $q$ is isotone we obtain

$$
\begin{aligned}
b=q\left(x, x_{1}, \cdots, x_{n}\right) & \geq q\left(x_{A}, x_{1}, \cdots, x_{n}\right) \\
& \geq q\left(x_{B},\left(x_{1}\right)_{B}, \cdots,\left(x_{n}\right)_{B}\right)=b_{B}=b,
\end{aligned}
$$

and thus $b=q\left(x_{A}, x_{1}, \cdots, x_{n}\right)$, as required.

We proceed similarly in the other three cases. This completes the proof of Lemma 2.

Lemma 3. Let $X$ be a generating set of $L$ satisfying $X \cap L_{1}=\varnothing$. If $\left\{x \mid x \in X, x_{A} \in A, x_{B} \in B\right\}=\varnothing$, then $X \cap(A \cup B)$ is a generating set of $L$.

Proof. By Lemma $1, T_{A}(X) \cup T_{B}(X)$ is a generating set of $L$. Since

$$
\begin{aligned}
& X=(X \cap A) \cup(X \cap B) \cup\left\{x \mid x \in X, x^{A} \in A \text { and } x^{B} \in B\right\}, \\
& T_{A}(X) \cup T_{B}(X)=(X \cap A) \cup(X \cap B)=X \cap(A \cup B),
\end{aligned}
$$

completing the proof.

Lemma 4. Let $X$ be a generating set of $L$ satisfying $X \cap L_{1}=\varnothing$. Then there is a generating set $T_{2} \subseteq A \cup B$ of $L$ satisfying $|X| \geq\left|T_{2}\right|$.

Proof. Represent $X$ in the form

$$
X=(X \cap A) \cup(X \cap B) \cup X_{1} \cup X_{2},
$$

where

$$
\begin{aligned}
& X_{1}=\left\{x \mid x \in X, x_{A} \in A, x_{B} \in B\right\}, \\
& X_{2}=\left\{x \mid x \in X, x^{A} \in A, x^{B} \in B\right\} .
\end{aligned}
$$

If $X_{1}=\varnothing$ then, by Lemma 3, we can set $T_{2}=X \cap(A \cup B)$. By duality, if $X_{2}=\varnothing$ we can set $T_{2}=X \cap(A \cup B)$. So assume that $X_{1} \neq \varnothing$ and $X_{2} \neq \varnothing$, and, say, $\left|X_{1}\right| \leq\left|X_{2}\right|$. Define 


$$
T_{2}=(X \cap A) \cup(X \cap B) \cup T_{A}\left(X_{1}\right) \cup T_{B}\left(X_{1}\right) .
$$

Observe that $\left|X_{1}\right| \geq\left|T_{A}\left(X_{1}\right)\right|$ and $\left|X_{2}\right| \geq\left|X_{1}\right| \geq\left|T_{B}\left(X_{1}\right)\right|$, hence

$$
\left|X_{1} \cup X_{2}\right| \geq\left|T_{A}\left(X_{1}\right) \cup T_{B}\left(X_{1}\right)\right| \text {, }
$$

and so $|X| \geq\left|T_{2}\right|$. Since $T_{A}\left(X_{2}\right)=T_{B}\left(X_{2}\right)=\not \varnothing, T_{A}(X) \cup T_{B}(X)=T_{2}$, a reference to Lemma 1 completes the proof.

Now we are ready to prove the theorem. Since $A \cup B$ generates $L$, $g(A)+g(B) \geq g(L)$ is trivial. To prove the converse, let $X$ be a generating set with $|X|=g(L)$. By applying Lemma 2 repeatedly we obtain a generating set $X^{\prime}$ satisfying $|X| \geq\left|X^{\prime}\right|$ and $X^{\prime} \cap L_{1}=\varnothing$. Since $|X|=g(L)$, therefore $\left|X^{\prime}\right|=g(L)$. Applying Lemmas 3 and 4 we obtain a generating set $X^{\prime \prime} \subseteq$ $A \cup B$ with $\left|X^{\prime}\right| \geq\left|X^{\prime \prime}\right|$. Again, $\left|X^{\prime \prime}\right|=g(L)$. By Lemma $1, T_{A}\left(X^{\prime \prime}\right)=X^{\prime \prime} \cap A$ generates $A$ and $T_{B}\left(X^{\prime \prime}\right)=X^{\prime \prime} \cap B$ generates $B$, so

$$
g(L)=\left|X^{\prime \prime}\right|=\left|X^{\prime \prime} \cap A\right|+\left|X^{\prime \prime} \cap B\right| \geq g(A)+g(B),
$$

concluding the proof of the main result.

Theorem 1. Let $\mathrm{K}$ be a nontrivial equational class of lattices and let $L \in \mathbf{K}$ be the free $\mathbf{K}$-product of $A$ and $B$. Then $g(L)=g(A)+g(B)$.

3. The most immediate consequence of Theorem 1 is the following.

Corollary 2. Let $\mathbf{K}$ be a nontrivial equational class of lattices and let $L \in \mathbf{K}$ be the free $\mathbf{K}$-product of the lattices $L_{i}$, $i \in I$. Then

$$
g(L)=\sum\left(g\left(L_{i}\right) \mid i \in I\right) .
$$

Proof. If $I$ is finite, then this follows by induction on $|I|$ from Theorem 1. If $I$ is infinite, then $g(L)=|L|$ and the conclusion is obvious.

Corollary 3. Let $\mathbf{K}$ be a nontrivial equational class of lattices. Let $n$ be a positive integer and let $L \in \mathbf{K}$ be n-generated. If $L$ is a free $\mathbf{K}$ product of $L_{i}$, $i \in I$, then $|I| \leq n$.

Proof. Indeed, by Corollary 2, $n \geq g(L)=\Sigma\left(g\left(L_{i}\right) \mid i \in I\right) \geq|I|$, where the last inequality is obtained from $g\left(L_{i}\right) \geq 1$.

Corollary 4. Under the conditions of Corollary 3, L is a free K-product of exactly $n$ lattices iff $L$ is $F_{\mathbf{K}}(n)$, that is, a free $\mathbf{K}$-lattice on $n$ generators.

Proof. If $L=F_{\mathbf{K}}(n)$, then $L$ is a free $\mathbf{K}$-product of $n$ copies of $F_{\mathbf{K}}(1)$. 
Conversely, if $L$ is $n$-generated and $L$ is a free $\mathbf{K}$-product of $L_{1}, \cdots, L_{n}$, then $n \geq g\left(L_{1}\right)+\cdots+g\left(L_{n}\right)$ by Corollary 3 and so $g\left(L_{1}\right)=\cdots=g\left(L_{n}\right)=1$, that is, $L_{1} \cong \cdots \cong L_{n} \cong F_{\mathbf{K}}(1)$, and $L \cong F_{\mathbf{K}}(n)$, as claimed.

4. There is a lattice situation to which our results apply that is more general than free $\mathbf{K}$-products.

Definition 5. The lattice $L$ is a pseudo-free product of its sublattices $L_{i}, i \in I$, iff $L$ is generated by $\bigcup\left(L_{i} \mid i \in I\right)$ and for each $i \in I$ there are homomorphisms

$$
\begin{aligned}
& x \rightarrow x_{L_{i}}, \quad L \rightarrow L_{i} \cup\{0\} \\
& x \rightarrow x^{L_{i},} \quad L \rightarrow L_{i} \cup\{1\}
\end{aligned}
$$

which satisfy

$$
\begin{gathered}
a=a_{L_{i}}=a^{L_{i}} \text { for all } a \in L_{i}, \\
a_{L_{i}}=0, \quad a^{L_{i}}=1 \text { for all } a \in L_{j}, j \neq i, j \in I .
\end{gathered}
$$

The simplest example of a pseudo-free product which is not a free $\mathbf{K}$ product is given in Figure 1; it is a pseudo-free product of the singleton $\{a\}$ with the two-element chain $\left\{b_{1}, b_{2}\right\}$.

It is easy to check that for $a \in L_{i}, b \in L_{j}, i \neq j, i, j \in I, a$ and $b$ are incomparable; indeed, if $a \leq b$, then $a=a_{L_{i}} \leq b_{L_{i}}=0$, a contradiction. Similarly, we can verify all the properties of free $\mathbf{K}$-products that were used in proving Theorem 1 of this note. In fact, the common refinement property for free $\mathbf{K}$-products proved in [3] relies only on the se properties. We obtain:

Theorem 6. Let the lattice $L$ be a pseudo-free product of $\left(A_{i} \mid i \in I\right)$ and of $\left(B_{j} \mid j \in J\right)$. Then $L$ is a pseudo-free product of $\left(A_{i} \cap B_{j} \mid i \in I\right.$, $\left.j \in J, A_{i} \cap B_{j} \neq \varnothing\right)$, and, for $i \in I, A_{i}$ is a pseudo-free product of $\left(A_{i} \cap B_{j}\right)$ $\left.j \in J, A_{i} \cap B_{j} \neq \varnothing\right)$.

Theorem 7. Let the lattice $L$ be a pseudo-free product of $A$ and $B$. Then $g(L)=g(A)+g(B)$.

Naturally, the consequences of Theorem 6 given in [3] and the corollaries of Theorem 7 given in $\$ 3$ also hold.

Finally, we would like to point out that the results of this note, as well as those of [3], generalize to some algebras other than lattices. All 


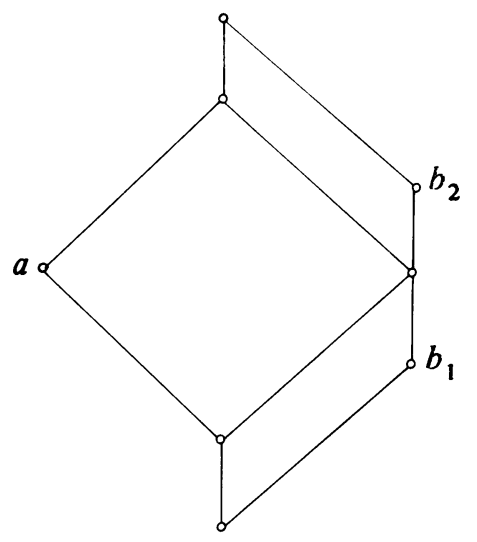

Figure 1

that is needed is that the algebras be partially ordered, that polynomials and homomorphisms be isotone, and finally that 0 and 1 can be adjoined in a manner permitting the introduction of covers. For instance, lattice ordered semigroups have all these properties, and therefore all the results of this note and of [3] apply to lattice ordered semigroups.

\section{REFERENCES}

1. G. Grätzer, Lattice theory: First concepts and distributive lattices, Freeman, San Francisco, Calif., 1971.

2. G. Grätzer, H.. Lakser and C. R. Platt, Free products of lattices, Fund. Math. 69 (1970), 233-240. MR 43 \#116.

3. G. Grätzer and J. Sichler, Free decompositions of a lattice, Canad. J. Math. (to appear).

4. B. Jónsson, Relatively free products of lattices, Algebra Universalis 1 (1971/72), 362-373. MR $46 \# 106$.

DEPARTMENT OF MATHEMATICS, UNIVERSITY OF MANITOBA, WINNIPEG, MANITOBA R3T 2N2, CANADA 